\title{
Comparación entre la prevalencia de ansiedad y depresión en las estudiantes del último año de medicina de dos universidades de México
}

\author{
Terrones Saldivar Ma del Carmen*, Rosas Cabral Alejandro*, Reyes Robles Martha Elena**
}

\begin{abstract}
Resumen
- Antecedentes. La ansiedad y la depresión tienen una preva- lencia que varía de acuerdo a la población estudiada. En los - estudiantes de medicina, la frecuencia de estos padecimientos - es elevada debido a diversos factores estresantes, caracterís- ticos de la misma carrera. Objetivo de investigación: Conocer y - comparar las prevalencias de depresión y ansiedad entre los - estudiantes del último año de la carrera de medicina de la Uni- versidad Autónoma de San Luis Potosí (UASLP) y de la Universi- dad Autónoma de Aguascalientes (UAA). Metodología. Estudio - comparativo, transversal y prospectivo. Se estudiaron a 56 y 65

- estudiantes del décimo semestre de la carrera de medicina de - la UAA y de la UASLP, respectivamente, inscritos en el semestre - enero-junio 20/3. Se evaluó, mediante la escala de Zung, la - depresión, y la de Hamilton, para la ansiedad. La participación - de los encuestados fue anónima y bajo consentimiento infor-

- mado. Resultados y conclusión. La prevalencia de depresión y - de ansiedad fueron muy similares entre los grupos de estudio - (I6 y 17\% para la depresión; y de 35 y 32\% para la ansiedad, - UAA y UASLP, respectivamente). No encontramos diferencias - estadísticamente significativas entre los dos grupos estudiados.

- Sin embargo, encontramos que la prevalencia de depresión y - ansiedad en las mujeres del grupo de la UAA fue más elevada - que en los hombres, de manera significativa $(p<0.007)$. Debi-

- do a la frecuencia elevada de la ansiedad entre los alumnos

- evaluados, es conveniente que se implementen estrategias de - intervención en ambas carreras de medicina. LUX MÉDICA AÑO

- 9, NÚMER0 28, SEPTIEMBRE-DICIEMBRE 2014, PP 13-21
\end{abstract}

Palabras clave: depresión, ansiedad, estudiantes medicina.

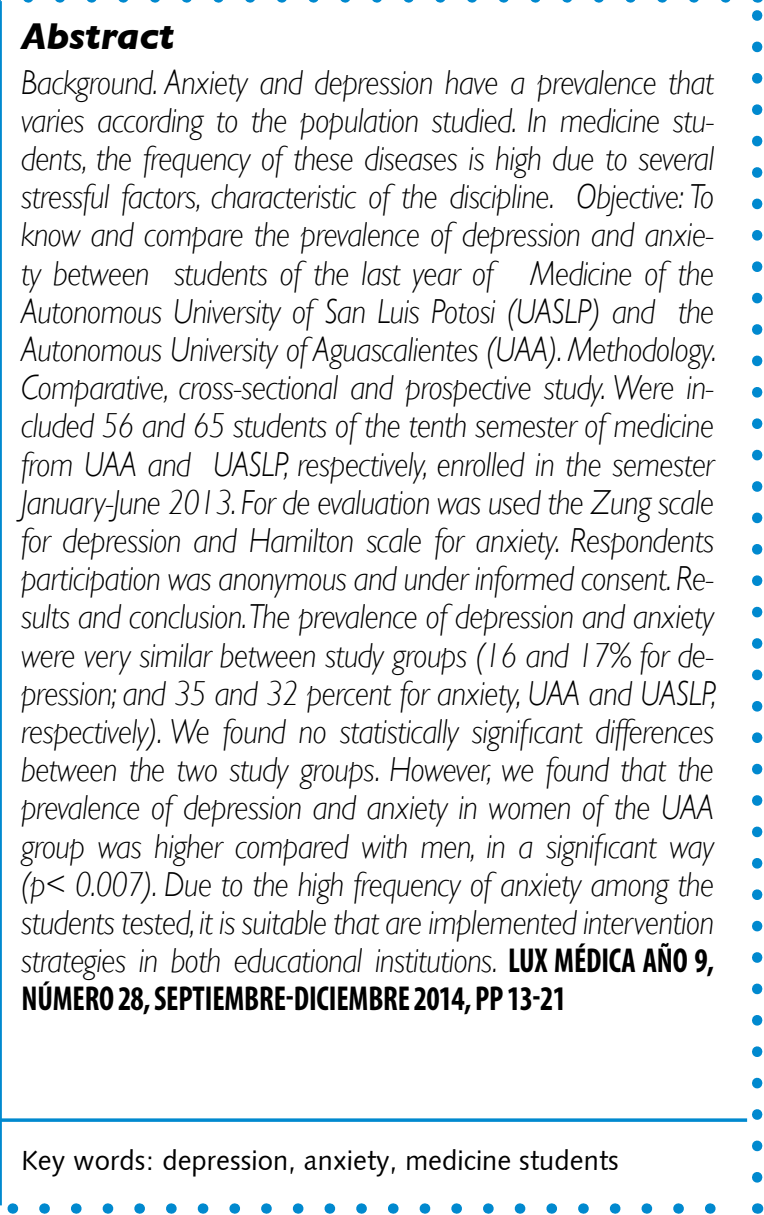

Profesores Investigadores del Centro de Ciencias de la Salud de la Universidad Autónoma de Aguascalientes ** Técnico Académico del Centro de Ciencias de la Salud de la Universidad Autónoma de Aguascalientes.

Fecha de recibido:2 de septiembre 2014

Fecha de aceptación: 23 de octubre 2014

Correspondencia: Dra en C Ma del Carmen Terrones Saldívar. Profesora investigadora del Departamento de Gíneco-Obstetricia y Pediatría del Centro de Ciencias de la Salud de la Universidad Autónoma de Aguascalientes. Edificio 107 planta alta. Avenida Universidad \#910 Ciudad Universitaria. Aguascalientes, Ags., México. Código Postal 20131. Teléfono +52(449)9108443. Correo electrónico mcterron@correo.uaa.mx

0




\section{Introducción}

La depresión y la ansiedad son trastornos mentales de alta prevalencia en el mundo y representan una carga social y económica al repercutir en la calidad de vida de las personas afectadas en su entorno laboral y social. Por lo anterior se considera como un tema de salud pública emergente. ${ }^{1}$ La depresión es un trastorno afectivo que se caracteriza por la presencia de tristeza, pérdida de interés o placer, sentimientos de culpa o falta de autoestima, trastornos del sueño o del apetito, sensación de cansancio y falta de concentración. $^{2}$ De acuerdo a cifras reportadas por la Organización Mundial de la Salud (OMS), la depresión afecta aproximadamente a 350 millones de personas en el mundo y es, además, una de las principales causas de discapacidad. ${ }^{3}$ Afecta las actividades diarias, ya que manifiestan sentimientos de inutilidad, culpa, tristeza y desesperanza. La depresión no es una moda, constituye un severo problema de salud pública que hoy por hoy afecta entre 12 y $20 \%$ a personas adultas, es decir, de entre 18 y 65 años. ${ }^{4-7}$ Por otro lado, la ansiedad se caracteriza por un intenso malestar interior que la persona no es capaz de controlar, esta última suele aparecer en algunos casos como síntoma de la depresión. En nuestro país, de acuerdo a lo reportado por la Encuesta Nacional de Epidemiología Psiquiátrica, la prevalencia de ansiedad fue de $14.3 \% .^{8}$

Existen reportes que han evaluado el comportamiento epidemiológico de estas enfermedades en poblaciones específicas. En particular, algunos autores han estudiado la distribución de estos trastornos mentales en los estudiantes de medicina, ya que el estudio de la medicina se caracteriza por una constante y creciente exigencia académica que demandan del estudiante esfuerzos de adaptación, ${ }^{9}$ partiendo de la premisa de que los estudiantes de medicina se desempeñan en un ambiente saturado de situaciones estresantes..$^{9-18}$ Osada y cols han reportado que los alumnos de medicina humana son especialmente sensibles, encontrándose niveles más elevados de estos síndromes en comparación a otros grupos académicos ${ }^{1}$, posiblemente explicado por diferentes factores entre los que se encuentra la elevada exigencia que representa para ellos, desde el mismo proceso de selección para ingresar a la carrera, para luego continuar con el rigor académico del área básica y clínica, aunado a la experiencia de las guardias que representan todo un desafío, para responder adecuadamente a las necesidades de los pacientes. Además existen otros factores que pueden potenciar la ansiedad y la depresión, como el cansancio, la falta de tiempo para dormir, la exigencia desmedida o inadecuada de algunos maestros y adscritos, entre otros. Algunos autores han reportado que la depresión repercute en el desarrollo académico y profesional de los estudiantes; ${ }^{19,20}$ otros la han asociado con la falta de empatía con el paciente y el deterioro de la calidad de la atención médica. ${ }^{21}$ Nosotros previamente reportamos que la prevalencia de depresión en 
estudiantes del último año de la carrera de medicina fue del $8 \%$, prevalencia que se incrementó significativamente cuando ingresaron al internado médico de pregrado. ${ }^{22}$ Recientemente, Fouilloux ${ }^{23}$ evaluó los probables casos de trastornos mentales en alumnos de los primeros años de la Facultad de Medicina y encontró que el $10.5 \%$ de los alumnos sufrían algún tipo de trastorno mental. Los síntomas más frecuentemente reportados por los alumnos fueron disminución de la concentración, estado de ánimo depresivo y ansiedad. ${ }^{23}$

Si aceptamos que los trastornos mentales pueden afectar no sólo el desempeño académico entre los estudiantes de la carrera de medicina, sino la atención que brindan a sus pacientes e incluso su propio cuidado, entonces comprenderemos la importancia de identificar la prevalencia de aquellos. Para la comparación pensamos en los estudiantes de medicina de la Universidad Autónoma de San Luis Potosí (UASLP) debido a que los planes de estudios de ambas carreras establecen materias y práctica clínica similares. En general los estudiantes del último año de la carrera de medicina llevan materias disciplinares de alta exigencia, además de cubrir práctica clínica y guardias nocturnas.

El objetivo de este trabajo fue conocer y comparar las prevalencias de depresión y ansiedad entre los estudiantes del último año de la carrera de medicina de la Universidad Autónoma de San Luis Potosí y de la Universidad Autónoma de Aguascalientes.

\section{| | | | | | | | | | | | | | | | | | | | | | | | | | | | | | | | | | | | | | | | | | | | | | | | | | | | | | | | | | | | | | | | | | | | | | | | | | | | | | | | | | | | | | | | | | | | | | | | | | | | | ||}

\section{Material y métodos}

Se realizó un proyecto descriptivo, comparativo, transversal y prospectivo. Quedaron incluidos, mediante muestreo por conveniencia, estudiantes del décimo semestre de medicina, 56 de la Universidad Autónoma de Aguascalientes y 65 de la Universidad Autónoma de San Luis Potosí, inscritos durante el semestre enero-junio 2013, sin importar el sexo o la edad. Se utilizó la escala de Zung para evaluar la depresión y la de Hamilton para evaluar la ansiedad. La participación de los encuestados fue anónima y bajo consentimiento informado. Los instrumentos fueron aplicados a los estudiantes en el salón de clases, se escogió el momento adecuado para que participaran en calma, sin la presión de tiempo ni de exámenes cercanos. Se dieron las instrucciones para el llenado de las escalas que tardaron en contestar en aproximadamente 30 minutos.

El instrumento autoaplicado de depresión de Zung evalúa algunos de los síntomas de la depresión. Es un instrumento validado, muestra unos aceptables índices de sensibilidad (85\%) y especificidad (75\%) cuando se aplica para detección de casos en población general y ha sido ampliamente utilizada con esta finalidad. El cuestionario consta de 20 afirmaciones, a las que se les da un puntaje que va de 1 a 4, dependiendo de la respuesta: 1 para la respuesta nunca o muy pocas veces; 2 para algunas veces, 3 para frecuentemente y 4 para siempre. En las preguntas 2, 5, 6, 11, $12,14,16,17,18$ y 20 se invierte el orden del puntaje. El puntaje para evaluar la severidad de los síntomas fue el siguiente: hasta 49 puntos, sin síntomas; de más de 50 pun- 
tos, depresión leve ó mínima; más de 60 puntos, depresión moderada y más de 70 puntos, depresión severa. No obstante que la escala de depresión de Zung no asegura por sí sola el diagnóstico de depresión, tiene la ventaja de que permite identificar el nivel de sintomatología de depresión que experimentan las personas evaluadas.

La escala de ansiedad de Hamilton, validada desde 1959, consta de 14 ítems, a los que se les da un puntaje de 0 a 4 dependiendo de la respuesta: 0 para ausente; 1 para leve; 2 para moderado; 3 para grave y 4 para muy grave. La interpretación del puntaje será: menos de 5 puntos, sin síntomas; de 6 a 14 puntos, ansiedad leve y más de 15 puntos, ansiedad moderada a grave. La ansiedad se considerará positiva cuando el puntaje total sea de 15 puntos o mayor. Los resultados se expresan en porcentajes, y para determinar diferencias entre los grupos se utilizó la prueba de $\chi^{2}$. Se consideró estadísticamente significativo cuando la $\mathrm{p}<0.05$.

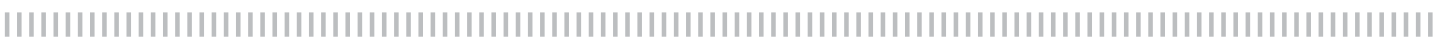

\section{Resultados}

Quedaron incluidos 121 estudiantes. Se encuestaron a 56 estudiantes de la UAA, con un promedio de edad de $22.6 \pm 1.9$ años, 29 hombres (51.7\%) y 27 mujeres
(48.3\%). Los resultados de las encuestas aplicadas a los 65 estudiantes de la UASLP mostraron un promedio de edad de $22.9 \pm 2.1$ años, 33 mujeres $(50.7 \%)$ y 32 hombres $(49.3 \%)$. (Tabla 1$)$.

\section{Tabla I}

Distribución por edad y sexo de los estudiantes del último semestre de Medicina de la UAA y de la UASLP. No hubo diferencias significativas.

\begin{tabular}{|lcc|}
\hline & Estudiantes UAA \\
$(\mathbf{n = 5 6 )}$ & $22.6 \pm 1.9$ & $\begin{array}{c}\text { Estudiantes UASLP } \\
\text { (n=65) }\end{array}$ \\
\hline Edad (años) Promedio \pm DS & $51 \%$ & $22.9 \pm 2.1$ \\
\hline Sexo femenino (porcentaje) & $(27 / 56)$ & $50.7 \%$ \\
\hline & $49 \%$ & $(33 / 65)$ \\
\hline Sexo masculino (porcentaje) & $(29 / 56)$ & $49.3 \%$ \\
\hline & & $(32 / 65)$ \\
\hline
\end{tabular}

Para el grupo de estudiantes de medicina de la UAA, encontramos una prevalencia de depresión de 16\% (9/56), con predominio de la depresión leve (6/9), seguida de la depresión moderada (3/9). En cuanto al sexo, encontramos que en 8 de los 9 estudiantes identificados con de- presión correspondieron al sexo femenino, solo un estudiante del sexo masculino fue identificado con depresión moderada. No se identificó ningún caso de depresión severa. Respecto a la ansiedad encontramos una prevalencia del 35\% (20/56) con una distribución de predominio en el sexo fe- 
menino $(n=18)$ sobre el sexo masculino $(n=2)$. En este grupo encontramos diferencias estadísticamente significativas cuando comparamos los resultados de depresión $(p<0.007)$ y ansiedad $(p<0.000)$ por sexo. (Tabla 2 y figura 1).

Para el grupo de estudiantes de medicina de la UASLP, encontramos una prevalencia de depresión de 17\% (11/65), con predominio de la depresión leve (10/11) y del sexo masculino (6/10), sólo hubo una estudiante con depresión moderada. No encontramos ningún caso de depresión severa. Respecto a la ansiedad, encontramos una prevalencia del $32 \%(21 / 65)$, con una distribución similar entre hombres $(n=10)$ y mujeres $(n=11)$. En este grupo no hubo diferencias significativas cuando se compararon los resultados de depresión y ansiedad por sexo ( $p>0.05)$. (tabla 2 y figura 1$)$.

\section{Tabla 2}

Distribución de las prevalencias de ansiedad y depresión, por severidad, en estudiantes del décimo semestre de la carrera de medicina de dos universidades públicas. No hubo diferencias significativas $(p>0.05)$

\begin{tabular}{|c|c|c|c|c|}
\hline & & $\begin{array}{l}\text { Estudiantes UAA } \\
\qquad(n=56)\end{array}$ & $\begin{array}{l}\text { Estudiantes UASLP } \\
\qquad(n=65)\end{array}$ & $\mathbf{p}$ \\
\hline \multirow[t]{4}{*}{ Ansiedad } & Moderada-severa & $20 / 56$ & $21 / 65$ & \\
\hline & Prevalencia de ansiedad & $35 \%$ & $32 \%$ & 0.69 \\
\hline & & $\begin{array}{l}\text { Estudiantes UAA } \\
\qquad(n=56)\end{array}$ & $\begin{array}{l}\text { Estudiantes UASLP } \\
\qquad(n=65)\end{array}$ & \\
\hline & Leve & $6 / 9$ & $10 / 11$ & \\
\hline \multirow[t]{4}{*}{ Depresión } & Moderada & $3 / 9$ & $1 / 11$ & \\
\hline & Severa & 0 & 0 & \\
\hline & Prevalencia de depresión & $16 \%$ & $17 \%$ & 0.089 \\
\hline & & $(9 / 56)$ & $(11 / 65)$ & \\
\hline
\end{tabular}

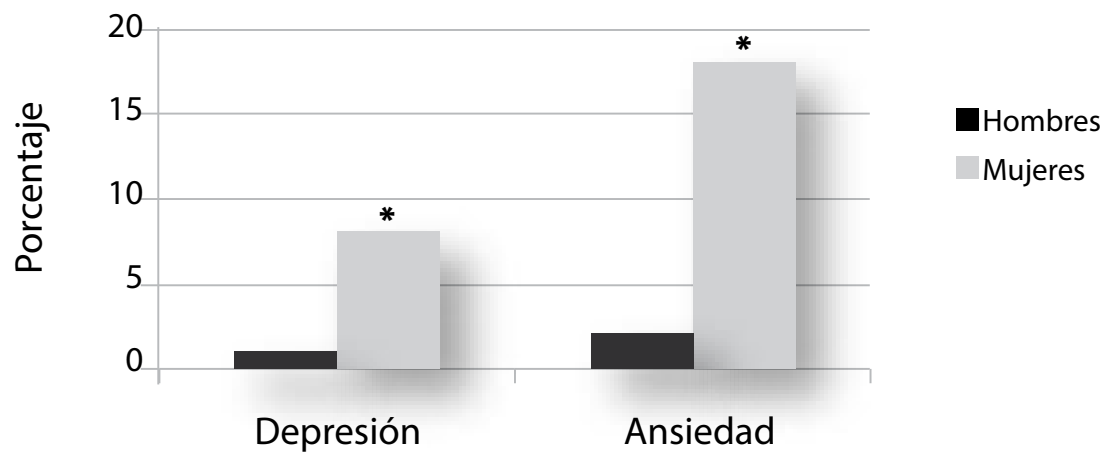

Figura 1. Distribución de la depresión y ansiedad, por sexo, entre los estudiantes del último año de medicina de la Universidad Autónoma de Aguascalientes. $(p<0.007)$ 
Cuando comparamos las prevalencias de depresión y ansiedad entre los estudiantes de la UAA y los de la UASLP $(16 \%$ vs
$17 \%$ y $35 \%$ vs $32 \%$, respectivamente), no encontramos diferencias significativas ( $p>0.05$ ) (figura 2)

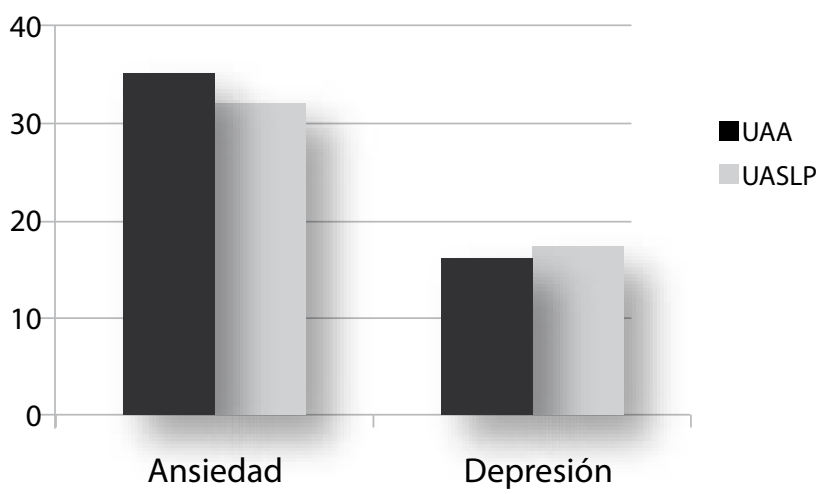

Figura 2. Distribución de la prevalencia de ansiedad y depresión evaluada, en estudiantes del décimo semestre de la carrera de medicina de dos universidades públicas. Las diferencias no fueron significativas.

\section{Discusión}

Los trastornos mentales son enfermedades que se caracterizan por producir alteraciones en el pensamiento, afecto, emociones y comportamiento acompañados de alteración en el funcionamiento del individuo afectado, además son entidades que se prolongan en el tiempo o tienen un carácter recurrente. ${ }^{24}$

Nuestros resultados mostraron una similitud de las prevalencias de depresión y ansiedad entre los estudiantes encuestados: $16 \%$ para los estudiantes de la UAA y $17 \%$ en los estudiantes de la UASLP (depresión), y de $35 \%$ y $32 \%$ respectivamente, para la ansiedad. La prevalencia para depresión de ambos grupos cae en el rango que ha reportado la OMS, sin que esto quiera decir que no representa un motivo de preocupación para las instituciones que forman a los futuros médicos. Por otra parte, la prevalencia de ansiedad encontrada es mayor a lo reportado por la Encuesta Nacional de Epidemiología Psiquiátrica $(14.3 \%)^{8}$. Podemos decir que aproximadamente uno de cada tres estudiantes de medicina que estudiamos presenta ansiedad moderada-severa. Sin embargo, cuando la comparamos con lo reportado por Celis, observamos que ellos encontraron una prevalencia para ansiedad casi el doble de la reportada en este trabajo (64.2\% en los estudiantes de primer año y de $57.8 \%$ al finalizar la carrera). ${ }^{25}$ La identificación de la ansiedad en los estudiantes de medicina ha sido reportada tanto en países occidentales como orientales y también en países desarrollados como en aquellos en vías de desarrollo, ${ }^{26-28}$ así mismo, se ha sugerido que la salud mental de los estudiantes de medicina se encuentra más deteriorada poco después de su ingreso a la carrera de medicina, esto es durante el primer año de la misma, y que estas condiciones continúan a través de todo su entrenamiento.

Otros autores han reportado que los estudiantes de medicina tienen una elevada prevalencia de ansiedad y depresión, con niveles de estrés psicológico consistentemente mayores que la población general y 
que sus pares por edad cursando otras carreras. $^{29}$

En la siguiente tabla (tabla 3) podemos observar diferentes resultados que nos permiten comparar nuestros resultados. Es de llamar la atención que existen diferentes prevalencias, unas mayores y otras menores a la encontrada en este trabajo, debido posiblemente a que las condiciones específicas varían de un entorno a otro, además de que no se utiliza un instrumento único para evaluar ansiedad y depresión.

\section{Tabla 3}

Prevalencias de depresión reportadas por diferentes autores.

\begin{tabular}{|c|c|c|}
\hline Observaciones & Prevalencia de depresión (\%) & Referencia \\
\hline \multirow[t]{2}{*}{272 estudiantes } & 31.6 & \\
\hline & (predominio del sexo femenino) & Hidalgo y cols, 2003. Argentina ${ }^{30}$ \\
\hline \multirow[t]{2}{*}{500 estudiantes } & 12.5 & \\
\hline & (predominio del sexo femenino) & Riveros y cols 2007, Perú31 \\
\hline \multirow[t]{2}{*}{165 estudiantes } & 28.6 & \\
\hline & & Ahmed Isra y cols, 2009. Dubai ${ }^{32}$ \\
\hline \multirow[t]{2}{*}{378 estudiantes } & 29.9 & \\
\hline & ( no diferencia por sexo) & Osada y cols, 2010, Perú ${ }^{1}$ \\
\hline \multirow[t]{2}{*}{481 estudiantes } & 8.6 & \\
\hline & ( no diferencia por sexo) & Al- Busaidi, 2011, Oman. ${ }^{33}$ \\
\hline \multirow[t]{2}{*}{1089 estudiantes } & $5.7-10.6$ & \\
\hline & ( no diferencia por sexo) & Quince y cols, 2012 U. Cambrige UK ${ }^{34}$ \\
\hline \multirow[t]{2}{*}{593 estudiantes (área de la salud) } & $12.3-16$ & \\
\hline & (predominio del sexo femenino) & Terrones y cols, 2014, UAA México ${ }^{35}$ \\
\hline \multirow[t]{2}{*}{259 estudiantes } & 8.77 & \\
\hline & (predominio del sexo femenino) & Agudelo Vélez, Colombia, $2008^{36}$ \\
\hline \multirow[t]{2}{*}{450 estudiantes } & 6.59 & \\
\hline & & Ibrahim Nahla, Arabia Saudita, $2013^{37}$ \\
\hline
\end{tabular}

Otro hallazgo interesante que encontramos es que en el grupo de estudiantes de la UAA, identificados con ansiedad y con depresión, observamos un predominio en el sexo femenino, condición que no encontramos en el grupo de estudiantes de la UASLP. Estos resultados son acordes a lo reportado por algunos autores, donde han publicado que entre estudiantes universitarios, la prevalencia en mujeres es mayor que en los hombres. ${ }^{30,31}$ Esta característica encontrada en las mujeres que estudian medicina en la UAA, debe ser motivo de investigación para tratar de identificar los principales factores que están influyendo en nuestras estudiantes.
No obstante, a pesar de que el abordaje del estudio de la depresión y sus causas en los estudiantes de medicina puede tornarse complejo, es importante no sólo acercarse al problema sino también implementar estrategias de apoyo para los estudiantes, ya que la OMS ha identificado al suicidio como una de las primeras diez causas de muerte a nivel mundial, de los cuales el 30\% está dado por estudiantes universitarios. ${ }^{22} \mathrm{La}$ depresión también puede repercutir, como lo señala Castillo y colaboradores (2010), en la disminución del desarrollo profesional y académico, el abuso de sustancias, la disminución de actitudes humanitarias y la falta de empatía con los pacientes. Por lo 
anterior, consideramos que el cuidado de la salud de los futuros médicos que atenderán a la población general es un imperativo ético evidente, en particular en lo referente a la salud mental. 17

A pesar de las limitaciones de este trabajo descriptivo, por la muestra reducida y la falta de aleatorización, aunado a que no proporciona información acerca de las causas de estos trastornos y sus consecuencias, los resultados nos obligan a reflexionar y proponer estrategias para el cuidado de la salud mental de los futuros médicos que atenderán a la población general.

\section{Conclusiones}

Nuestro estudio encontró que en los alumnos de medicina incluidos, la depresión tiene una prevalencia semejante a la de la población general. Para la ansiedad, encontramos una alta frecuencia en los grupos estudiados. No encontramos diferencias significativas al comparar la prevalencia de depresión y de ansiedad entre los grupos. El predominio del sexo femenino fue estadísticamente significativo para el grupo de la UAA. Se destaca en los dos grupos problemas de salud mental que debieran ser atendidos durante su permanencia en la institución educativa. Es conveniente continuar con esta línea de investigación para abordar posibles causas y proponer programas de intervención.

\section{Bibliografía}

1. Osada J, Rosas M, RosalesC, Vega-Dienstmaier JM. Sintomatología ansiosa y depresiva en estudiantes de medicina. Rev Neuropsiquiatr, 2010;73(1):15-19

2. Organización Mundial de la Salud. Disponible en http://www.who.int/topics/depression/es/ .

3.. Organización Mundial de la Salud. Disponible en http://www. paho.org/hq/index.php?option =com content\&view $=$ article\&id $=7305 \% 3$ Adia-mundialde-la-salud-mental-la-depresion-es-el-trastornomental-mas-frecuente- $\&$ catid $=740 \% 3$ Anewspress-releases \&ltemid $=1926 \&$ lang $=\mathrm{es}$.

4. Secretaría de Salud. Depresión y suicidio en México Secretaría de Prevención y Promoción de la Salud. Disponible en http://www.spps.gob.mx/ avisos/869-depresion-y-suicidio-mexico.html, consultado el 10 de enero 2014

5. Medina-Mora ME, Borges G, Lara C, Benjet C, Blan$\mathrm{co} J$ et al. Prevalence, service use, and denmographic correlates of 12-month DSM-IV psychiatric disorders in Mexico results fron the Mexican national Comorbidity Survey. Psychol Med. 2005;35(12):177731783.

6. Wagner FA, González-Forteza C, Sánchez-García S, García-Peña C, Gallo JJ. Enfocando la depresión como problema de salud pública en México. Salud Mental 2012;35(1)3-13.
7. Flores-Ocampo $R$, Jiménez-Escobar SD, PérezHernández S, Ramírez-Serrano PB, Vega- Valero CZ. Depresión y Ansiedad en Estudiantes Universitarios. Revista Electrónica de Psicología Iztacala 2007;10(2):94-105. Disponible en http://www. revistas.unam. $\mathrm{mx} /$ index. php/repi/article/viewFile/19112/18139, consultada el 10 de enero 2014.

8. Encuesta Nacional de Epidemiología Psiquiátrica 2003-2010. Disponible en http://www.inprf.gob. $\mathrm{mx} / \mathrm{psicosociales/archivos/encuestaepidemiologia.}$ pdf

9. Perales A, Sogi C, Morales R. Estudio comparativo de salud mental en estudiantes de medicina de dos universidades estatales peruanas. Anales de la Faculta de Medicina 2003;64(4):239-246.

10. Dyrbye LN, Thomas MR, Massie FS, Power DV, Eacker A, Harper W, Durning S, Moutier C, Szydlo DW Novotny PJ, Sloan JA, Schanafelt TD. Burnout and suicidal ideation among U.S. medical students. Ann Intern Med. 2008; 149(5):334-341.

11. Dyrbre LN, Harper W, Duming SJ, Moutier C, Thomas MR, Massie FS, Eacker A, Power DV, Szydlo DW Sloan JA, Shanafelt TD. Patterns of distress in US medical students. Med Teach. 2011; 33(10):834-838.

12. Joffre-Velázquez VM, Martínez-Perales G, GarcíaMaldonado G, Sánchez-Gutiérrez de Lara L. Depresión en estudiantes de medicina. Resultados de la aplicación del inventario de depresión de Beck en 
su versión de 13 ítems. Revista Argentina de Clínica Neuropsiquiátrica, 2007; 16(14):86-93.

13. Sen S, Kranzler HR, Krystal JH, Speller H, Chan G, Gelernter J, Guille C. A prospective cohort study investigating factors associated with depression during medical internship. Arch Gen Psychiatr. 2010 67(6):557-565.

14. Gaviria S, Rodríguez MA, Alvarez T. Calidad de la relación familiar y depresión en estudiantes de medicina de Medellín, colombia. Rev Chil Neuro-psiquiatr. 2002;40(1):41-46

15. Nogueria-Martins LA, Fagnani Neto $R$, Macedo PCM, Cítero VA, Mari JJ. The mental health of gradúate students at the Federal University os Sao Paulo: a preliminary report. Braz J Medi Biol Res 2004;37(10):1519-1524

16. Dahli $M$, Joneborg $N$, Runeso B. Stress and depression among medical students: a cross-sectional study Med Educ 2005;39(6):594-604

17. Castillo-Vilca MJ, Prado-Mendoza CJ, Vega-Dienstmaier JM. Prevalencia de depresión en estudiantes del quinto año de medicina de una universidad privada de Lima. Rev Neuropsiquiatr, 2010;73(1):9-14

18. Balanza Galindo S, Morales Moreno I, Guerrero Muñoz J, Conesa Conesa A. Fiabilidad y validez de un cuestionario para medir en estudiantes universitarios la asociación de la ansiedad y depresión con factores académicos y psicosociofamiliares durante el curso 2004-2005. Rev Esp Salud Pública, 2008;82:189200

19. Lugo M, Lara C, González JE, Granadillo D. Depresión, ansiedad y estrés en estudiantes de medicina del área básica y clínica, su relación con el índice de lateralización hemisférica cerebral y el rendimiento académico. Universidad de Carabobo año 19992000. Archivos Venezolanos de Psiquiatría y Neurología, 2004;50(103):21-29.

20. Jara D, Velarde H, Gordillo G, Guerra G, León I, Arroyo $C$, Figueroa $M$. Factores influyentes en el rendimiento académico de estudiantes del primer año de medicina. An Fac Med, 2008;69(3):193-197.

21. Shanafelt TD, Bradley KA, Wipf JE, Back AL. Burnout and self-reported patient care in an internal medicine residency program. Ann Intern Med 2002;136:358367

22. Terrones-Saldívar MC, Rosas-Cabral A, García-Huízar P, Moreno-Castanedo H, Prieto-Macías J, ReyesRobles ME. Comparación de los niveles de ansiedad y depresión entre estudiantes del último año de la carrera de medicina de la UAA e internos de pregrado del estado de Aguascalientes. Lux Médica 2010:5(15): 9-14

23. Fouilloux-Morales $C$, Barragán-Pérez $V$, Ortiz-León $S$, Jaime-Medrano A, Urrutia-Aguilar MR, Guevara-Guzmán R. Síntomas depresivos y rendimiento escolar en estudiantes de Medicina. Salud Mental 2013;36:59-65

24. Bohórquez Peñaranda AP. Prevalencia de depresión y de ansiedad según las escalas de Zung, y evaluación de la asociación con el desempeño académico en los estudiantes de medicina de la Pontificia Universidad Javeriana. (Tesis). Consultada el 10 de ene- ro 2014 y disponible en http://repository.javeriana. edu.co/bitstream/10554/442/1/med3.pdf

25. Celis J, Bustamante $M$, Cabrera D, Cabrera $M$, Alarcón W, Monge E. Ansiedad y Estrés Académico en Estudiantes de Medicina Humana del Primer y Sexto Año. Anales de la Facultad de Medicina 2001; 622530. Disponible en: http://www.redalyc.org/articulo. oa?id=37962105. Consultado el 13 de enero de 2014.

26. Lupo MK, Straus RD. Religiosity, anxiety and depression among Israeli medical students. Isr Med Assoc J. 2011;13(10):613-618

27. Imam SN, Sagib A, Alam E. Prevalence of anxiety and depression among medical students of private university. J Pak Med Assoc. 2003;53(2);44-47.

28. Samaranayake CB, Fernando AT. Satisfaction with life and depression among medical students in Auckland, New Zealand. N Z Med J. 2011;124(1391):12-17.

29. Dyrbye LN, Thomas MR, Shanafelt TD. Systematic review of depression, anxiety, and other indicators of psychological distress among U.S. and Canadian medical students. Acad Med 2006; 81(4):354-373.

30. Hidalgo EJ, Cendali JM, Marcelo A, Cuenca JGD, Kappaun BN. Prevalencia de depresión en estudiantes de 5tp año de la Facultad de Medicina de la UNNE 2003. Disponible en http://www.unne.edu.ar/unnevieja/Web/cyt/com2004/3-Medicina/M-065.pdf

31. Riveros $M Q$, Hernández $H$, Rivera J. Niveles de depresión y ansiedad en estudiantes universitario de Lima Metropolitana. Revista IIPSI, 2007;10(1):91102

32. Ahmed Isra, Banu Haseena, Al-Fageer Reem, AlSuwaidi Reem. Cognitive emotions: Depression and anxiety in medical students and staff . Journal of Critical Care, 2009;24:e1-e18

33. Al-Busaidi Zakiya, Bhargava Kam.lesh, Al-Ismaily Aida, Al-Lawati Hadia, Al-Kindi Rahma, Al- Shafaee Mohammad, Al-Maniri Abddullah. Prevalence of Depressive Symptoms among University Students in Oman. OmAN Medical Journal, 2011;26(4):235239.

34. Quince TA, Wood DF, Parker RA, Benson J. Prevalence and persistence of depression among undergraduate medical students: a longitudinal study at one UK medical school. BMJ Open 2012;00:e001519. Doi:10.1136/bmjopen-2012-001519

35. Terrones-Saldívar MC, Ruiz Esparza-Mota JA, Rosas-Cabral A, Reyes-Robles ME, Prieto-Macías J. Prevalencia de depresión en estudiantes de algunas carreras del Centro de Ciencias de la Salud de la Universidad Autónoma de Aguascalientes. Lux Médica,2014;9(26):15-19

36. Agudelo-Vélez DM, Casadiegos-Garzón CP, Sánchez-Ortiz DL. Características de ansiedad y depresión en estudiantes universitarios International Journal of Psychological Research, 2008;1(1):34-39

37. Ibrahim N, Al-Kharboush D, El-Khatib L, Al-Habib A, Asali D. Prevalence and predictors of anxiety and depression among female medical students in King Abdulaziz University, Jeddah, Saudi Arabia. Iranian J Publ Health , 2013;42(7):726-736 